\title{
LabView-based Simulator for PV Modules Energy Efficiency Optimization
}

\author{
Szász Csaba \\ Department of Electrical Machines and Drives \\ Technical University of Cluj \\ Csaba.Szasz@emd.utcluj.ro
}

\begin{abstract}
Due to the rapid depletion of traditional fossil energy resources the optimal utilization of renewable resources such as the solar energy becomes a leading task in power engineering. In recent years photovoltaic energy-linked technologies has reached major advances in order to achieve improved energy efficiency conversion rates. In this endeavor, a huge amount of high quality research effort is dedicated worldwide. This paper is focused to present the design and implementation steps of a LabView-based toolkit aimed to modeling and simulates solar photovoltaic $(P V)$ cells in a wide range of operation conditions. By using its well known mathematical model, it has been conceived a versatile and userfriendly simulator well suitable to study and evaluate energy efficiency conversion processes. In order to maximize the energy conversion efficiency the maximum power point tracking (MPPT) algorithm has been implemented. The virtual instrument (VI) developed in the graphical programming language allows fast and convenient monitoring of $P V$ cell output magnitudes and plots the I-U and P-U diagrams under different operation conditions. These curves have been compared then with the diagrams provided by the PV module manufacturer. The obtained results are in good agreement with the manufacturer's catalogue data.
\end{abstract}

Keywords-PV cell; energy efficiency; LabView model; virtual instrument; energy efficency.

\section{INTRODUCTION}

As it is well known, the important technological improvements reached mostly in the last decade in photovoltaic energy generation allow its widespread utilization in renewable energy applications. Solar energy is one of the most important renewable energy resources available abundantly on the earth. It is a theoretically inexhaustible and cheap source, while the electricity produced is clean (ecological) and silent. Nowadays the vast majority of solar energy resources are obtained by using photovoltaic systems that converts the solar light energy in electrical current during a process named photovoltaic effect. PV systems behave in an extraordinary and useful way: produce no pollution while producing electricity, are long lasting, no require fuels in their operation, can operate at moderate temperatures, has low noise, require little maintenance due to absence of any mechanical moving parts, have a rapid response, and can be made from silicon which is the second most abundant element in the earth's crust. Additionally, excepting the hybrid systems they contain no fluids or gases that can leak out and are completely eco-friendly [1, 2]. Last technological advances enable the PV cells utilization in a wide variety of applications ranging from microwatts order small scale remote applications to high power plants with megawatts of power. Therefore, they are also are recommended on decentralized or dispersed (on-site) installation, where the interconnection of high number PV cells strongly influences the global performances of the entire plant. In this sense a huge amount of worldwide research activity is dedicated to gain further improvement in solar cells cost, reliability and energetic efficiency $[3,4,5,6]$.

However, beside their immense advantages they also exhibit several important limitations or disadvantages. One of these is linked with their limited energy conversion efficiency. Despite of the fact that PV cell devices are commercialized in a wide range of semiconductor material products (mostly with nonocrystalline or polycrystalline silicon substrates), even the most energy efficient monocrystalline PV cells do not exceed around $14-16 \%$ conversion rates [6]. One other disadvantage is that the output electrical magnitudes (voltage and current) of a PV cell strongly depend up on external environmental conditions. Such parameters are the solar irradiation intensity, environmental temperature (weather conditions), isolation, or the cell sun tracking angle if the module is not fixed. Therefore, any change in atmospheric conditions and fluctuation in nature has an important effect regarding the delivered output power of the solar cell, increasing or decreasing it significantly. Obviously, PV cell manufacturers provide datasheets for their modules measured in conditions referred as standard test conditions (STC). However, the STC conditions are difficult to meet in real operation state or rarely occur outdoors. So, the utility of these datasheets in demanding computations or energy evaluation calculus represents a controversial issue.

When the PV modules behaviors and energy efficiency are analyzed their I-U and P-U characteristics also should be considered. Both are strongly nonlinear and due to the complex relationships between the output current and voltage, respectively output power and voltage, it varies with the solar irradiation intensity and environmental temperature. Closely studying these curves it is possible to observe that is a single point in which the PV module delivers the maximum output power and operates with the highest energy efficiency. 
In the related scientific literature this point is named as the Maximum Power Point (MPP). Therefore, in order to obtain the highest amount of energy quantity from a given cell it is essential to continuously track this maximum. A wide range of MPP tracking algorithms have been designed and implemented in international references $[6,7,8,9,10,11,12]$. Of course, there is not enough room to perform an exhaustive survey of all these high quality contributions (and also do not covers the scope of this paper). However, some of these will be referred and in discussed the followings to serve as an adequate theoretical background for designing and implementing a LabView-based simulator. The main scope of this development is to create an user-friendly and versatile toolkit to simulate PV modules energy efficiency in various operating conditions. Such a modeling and simulation program may become a useful research instrument to perform thorough PV cell efficiency investigations and to find other novel solutions regarding the MPP tracking problem.

\section{PV CELl MATHEMATICAL MODEL}

Theoretically, PV cells are devices which convert sunlight directly to electrical energy by generating a photovoltaic current within a thin layer of semiconductor structure embedding $p$ - $n$ junctions. In essence, when exposed to sunlight photons are absorbed by the semiconductor atoms, freeing electrons from the negative layer which flows then to the positive layer generating an electric current which is directly proportional to solar irradiation. Upon these main theoretical considerations accurate equivalent electrical circuit models has been developed in the related scientific literature $[5,6]$. Perhaps the most used among these to predict energy production in PV cells is the circuit configuration shown in figure 1 , where $R_{s}$ represents the equivalent series resistance and $R_{p}$ the parallel resistance. The above resistances are used because the PV cells are not ideal $p-n$ junctions and have power losses. The $R_{s}$ resistance is very small and arises from the ohmic contact between the metal and semiconductor internal resistance, respectively the $R_{p}$ resistance is very large and represents the surface quality along the module's periphery. In ideal case, $R_{s}=0$ and $R_{p}=\infty$ [8]. This model is a more practical one, which is widely used in studies and provides sufficient accuracy for most applications [5, 6, 7].

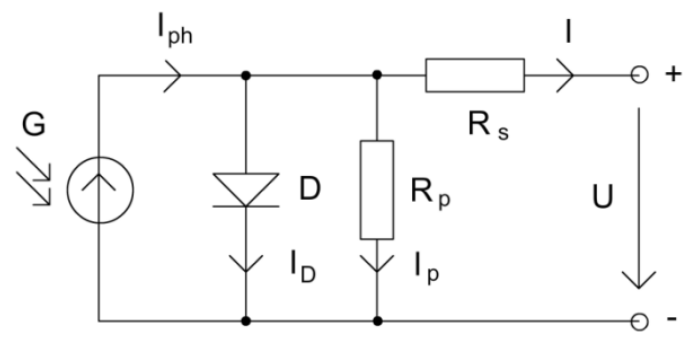

Fig. 1. The PV cell model with a single-diode, series and parallel resistance

Writing the Kirchhoff theorems for this equivalent circuit results the following equation:

$$
I=I_{p h}-I_{D}-I_{P}=I_{p h}-I_{0} \cdot\left\lfloor e^{\frac{q \cdot\left(U+I \cdot R_{S}\right)}{n \cdot k \cdot T}}-1\right\rfloor-\frac{U+I \cdot R_{S}}{R_{p}} .
$$

where $I_{p h}$ represents the photovoltaic current generated by the incidence of light, $I 0$ is the diode reverse bias saturation current $[A], \mathrm{U}$ is $q=1,6 \cdot 10^{-19} \mathrm{C}$ is the electron charge, $k=1,38 \cdot 10^{-23} \mathrm{~J} / \mathrm{K}$ is the Bolzmann constant, $n$ the diode ideality factor ( $n=2$ for silicon diodes), and $T$ is the temperature of the $p$-n junction $(K)$. The equation (1) represents the I-U output characteristics of the solar PV cell.

The cell operating temperature $T$ can be calculated as follows $[7,8]$ :

$$
T=\frac{\left(T_{N}-20\right)}{0.8} \cdot G+T_{A}
$$

where $T_{A}$ represents the ambient temperature $(K), G$ is the solar irradiance intensity $\left(\mathrm{W} / \mathrm{m}^{2}\right)$, and $T_{N}$ is the nominal operating cell temperature $(K)$ given in the manufacturer's datasheet. The mathematical expression of the photocurrent is written with the equation $[7,8]$ :

$$
\begin{aligned}
I_{p h}=I_{S N}\left[1+\mu_{I S C} \cdot\left(T-T_{N}\right)\right]= \\
=\frac{G}{G_{N}} \cdot I_{S C} \cdot\left[1+\mu_{I S C} \cdot\left(T-T_{N}\right)\right]
\end{aligned}
$$

In the above equation $G_{N}$ is the solar irradiation intensity at standard test conditions (STC: $G=1000 \mathrm{~W} / \mathrm{m}^{2}, T=25^{\circ} \mathrm{C}$ ), $I_{S N}$ is the nominal short-circuit current $(A), I_{S C}$ is the short-circuit current (A), respectively $\mu_{I s c}$ is the cell short-current coefficient $(A / K)$. The reverse bias saturation current can be expressed by the equation $[7,8]$ :

$$
I_{0}=\frac{I_{S C}}{e^{\frac{q \cdot U_{O C}}{n \cdot k \cdot T \cdot N_{S}} \cdot\left(1+\mu_{O C} \cdot\left(T-T_{N}\right)\right)}-1}
$$

where $U_{o c}$ is the open circuit voltage $(U)$ and $\mu_{o c}$ is the cell open circuit voltage temperature coefficient $(U / K)$.

\section{PV MODULE MATHEMATICAL MODEL}

In order to generate the required output current and output voltage levels the PV cells are connected in series and parallel configurations forming a PV module. When a number of $N_{s}$ solar cells are connected in series to build up a module the relationship (1) changes as follows [13]:

$$
I_{M s}=I_{p h}-I_{0} \cdot\left\lfloor e^{\frac{q \cdot\left(\frac{U_{M s}}{N_{s}}+I_{M s} \cdot R_{S}\right)}{n \cdot k \cdot T}}-1\right\rfloor-\frac{U_{M s}+I_{M s} \cdot N_{s} \cdot R_{s}}{N_{s} \cdot R_{p}}
$$

where $I_{M s}$ and $U_{M s}$ represents the serial module output current, respectively output voltage. Obviously, the above equation can be generalized to an arbitrary number of PV cells in series. If such serial configurations are connected in parallel in $N_{p}$ strings, with the output current $I_{M s p}$ and output voltage $U_{M s p}$, is found the following equation [13]: 


$$
\begin{aligned}
I_{M s p}= & N_{p} \cdot I_{p h}-N_{p} \cdot I_{0} \cdot\left\lfloor e^{\frac{q \cdot\left(\frac{U_{M s p}}{N_{S}}+I_{M s p} \cdot \frac{R_{S}}{N p}\right)}{n \cdot k \cdot T}}-1\right\rfloor- \\
& -\frac{U_{M s p}+I_{M s p} \cdot \frac{N_{S}}{N_{p}} \cdot R_{S}}{\frac{N_{S}}{N_{p}} \cdot R_{p}} .
\end{aligned}
$$

By using the substitutions $I_{M s p}=I, U_{M s p}=U$ results the simplified relationship:

$$
I=N_{p} \cdot I_{p h}-N_{p} \cdot I_{0} \cdot\left\lfloor e^{\frac{q \cdot\left(\frac{U}{N_{S}}+I \cdot \frac{R_{S}}{N_{p}}\right)}{n \cdot k \cdot T}}-1\right\rfloor-\frac{U+I \cdot \frac{N_{S}}{N_{p}} \cdot R_{S}}{\frac{N_{S}}{N_{p}} \cdot R_{p}} . \text { (7) }
$$

The above equation represents the mathematical model of the PV module shown below in figure 2.

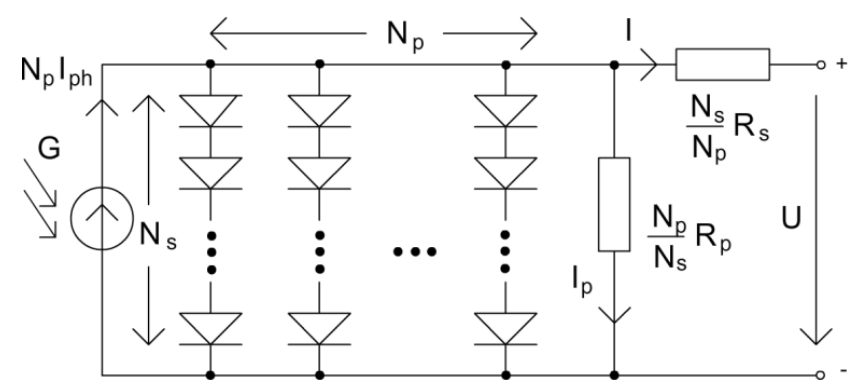

Fig. 2. PV module equivalent circuit

However, there the main drawback is that the equation (7) cannot be solved by using analytical methods. To overcome this bottleneck some authors uses the numerical NewtonRapson's method [8], others emphasize the solution using the approximation based on the Taylor series [7]. This last solution ensures easier and faster calculation with acceptable tolerance of precision. The basic idea of the method is relatively simple. Any continuous function with the form of $f(x)=e^{x}$ can be approximated with a Taylor series like:

$$
f(x)=e^{x}=\sum_{n=0}^{\infty} \frac{x^{n}}{n !}=1+x+\frac{x^{2}}{2 !}+\frac{x^{3}}{3 !}+\cdots
$$

Therefore, if the first order approximation is considered for the above function results that:

$$
e^{x} \cong 1+x
$$

Toward, assuming that $\mathrm{R}_{\mathrm{p}}=\infty$ and replacing $\mathrm{a}=\mathrm{q} / \mathrm{N}_{\mathrm{s}} \mathrm{nkT}$, the relationship (7) simplifies as follow:

$$
I=N_{p} \cdot I_{p h}-N_{p} \cdot I_{0} \cdot\left\lfloor e^{a \cdot U} \cdot e^{a \cdot \frac{N_{S} \cdot R_{S} \cdot I}{N_{p}}}-1\right\rfloor .
$$

By using the approximation results:

$$
e^{a \cdot \frac{N_{S} \cdot R_{S} \cdot I}{N_{p}}} \cong 1+a \cdot \frac{N_{S} \cdot R_{S} \cdot I}{N_{p}}
$$

$$
I=N_{p} \cdot I_{p h}-N_{p} \cdot I_{0} \cdot\left\lfloor e^{a \cdot U} \cdot\left(1+a \cdot \frac{N_{s} \cdot R_{S} \cdot I}{N_{p}}\right)-1\right\rfloor,
$$

from where

$$
I=\frac{N_{p} \cdot I_{p h}-N_{p} \cdot I_{0} \cdot\left(e^{a \cdot U}-1\right)}{1+a \cdot N_{S} \cdot R_{S} \cdot e^{a \cdot U}}
$$

The equation (13) expresses the I-U output characteristics of the PV module from figure 2.

\section{THE MAXIMAL POWER POINT TRACKING ALGORITHM}

As it is well known, a typical PV module converts only less than half of incident solar irradiation into electrical energy. In order to improve the efficiency of this energy conversion process basically two main procedures are available. The first refers to a solution relying on a mechanical tracking system suitable to orienting the PV module in such a direction as to receive the maximal solar radiation intensity in each moment. Of course, this solution looks feasible only in case when the captured additional energy amount is significantly higher than the energy quantity spent for the mechanical tracking system operation. The second one is to use the Maximum Power Point Tracking (MPPT) technique on the P-U output characteristics of the solar panel. In international references a great amount of high quality solutions have been proposed to implement this method [6, 7, $8,10]$. In fact, this procedure means the electrical tracking of the maximum power point, by continuously changing the equivalent load impedance to become equal with the source electrical circuit output impedance (Thevenin's theorem). However, the basic idea of these solutions is provided by mathematics, expressing that a continuous function $y=f(t)$ reaches its maximum when $d y / d t=0$. In this particular case, the output power $P$ becomes maximal when $d P / d U=0$. Among these MPPT algorithms perhaps one of the most used is the incremental conductance method. The operation principle of this solution is shown in figure $3[7,10]$.

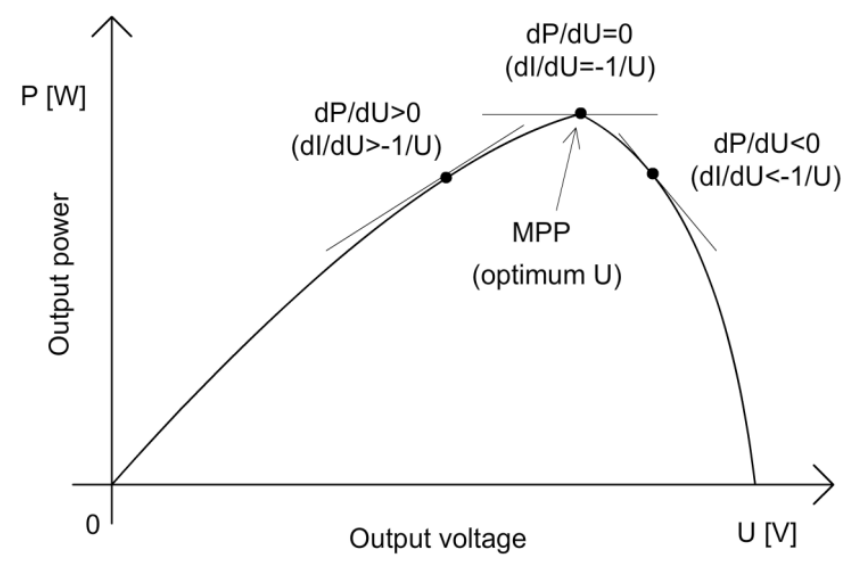


Fig. 3. The incremental conductance method

The mathematical description of the incremental conduction method is as follow:

$$
\frac{d P}{d U}=\frac{d(U \cdot I)}{d U}=\frac{d U \cdot I}{d U}+\frac{U \cdot d I}{d U}=I+U \cdot \frac{d I}{d U}
$$

When $d P / d U=0$ it is reached the MPP, therefore:

$$
d I / d U=-I / U .
$$

Left of MPP $d P / d U>0$ and $d I / d U<-I / U$, respectively right of MPP $d P / d U<0$ and $d I / d U<-I / U$. Obviously, it is not difficult to observe that the method utilizes the concept of "hill climbing", in which the slope of the P-U characteristics is zero at the maximum power point, positive at the left side and negative at the right side of the shape. The corresponding algorithm implementing the above ranked mathematical relationships is expressed in figure 4.

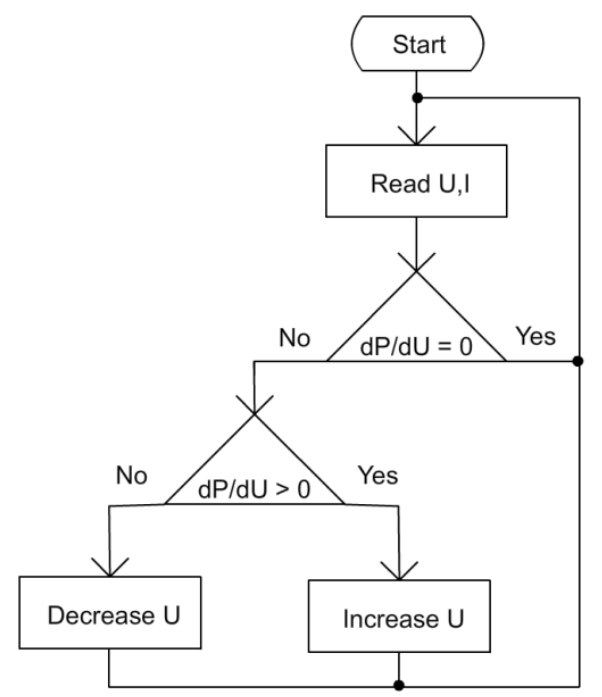

Fig. 4. The incremental conductance algorithm

In international references also it is mentioned that the incremental conductance method it is considered the most advantageous among the MPPT algorithms because it is easy to implement, is efficient with high execution speed, and it can identify and calculate the exact perturbation direction for the output voltage of PV panels [7].

\section{THE PV MODULE LABVIEW SIMULATION}

In order to validate the LabView model of the PV module it has been considered the solar panel unit 200Wp EGM 200, Mono (figure 4), manufactured by the EGing Photovoltaic Technology Co.,Ltd.

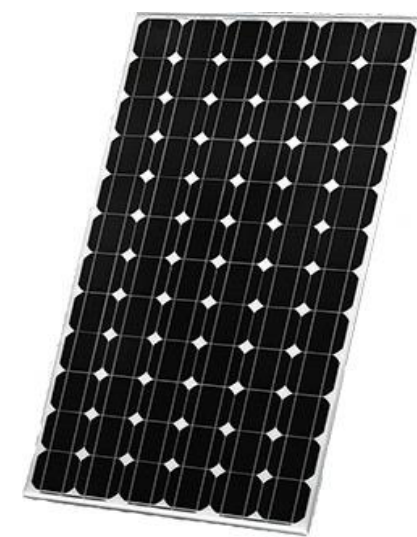

Fig. 5. The PV module 200Wp EGM 200, Mono-crystal This product has the manufacturer catalogue datasheet given next in Table 1 .

TABLE I. PV MANUFACTURER TECHNICAL SPECIFICATIONS

\begin{tabular}{|l|c|c|}
\hline \multicolumn{1}{|c|}{ Parameter } & Symbol & Value \\
\hline Peak power & $\mathrm{P}_{\max }[\mathrm{W}]$ & 200 \\
\hline Max. power current & $\mathrm{I}_{\mathrm{m}}[\mathrm{A}]$ & 5,52 \\
\hline Max. power voltage & $\mathrm{U}_{\mathrm{m}}[\mathrm{V}]$ & 36,25 \\
\hline Short circuit current & $\mathrm{I}_{\mathrm{sc}}[\mathrm{A}]$ & 5,86 \\
\hline Open circuit voltage & $\mathrm{U}_{\mathrm{oc}}[\mathrm{V}]$ & 44,61 \\
\hline Temp. co-efficient for $\mathrm{P}_{\max }$ & $\mathrm{K}_{\mathrm{pm}}\left[\mathrm{W} /{ }^{\circ} \mathrm{C}\right]$ & -0.86 \\
\hline Temp. co-efficient for $\mathrm{U}_{\mathrm{oc}}$ & $\mathrm{K}_{\mathrm{oc}}\left[\mathrm{V} /{ }^{\circ} \mathrm{C}\right]$ & -0.14498 \\
\hline Temp. co-efficient for $\mathrm{I}_{\mathrm{sc}}$ & $\mathrm{K}_{\mathrm{sc}}\left[\mathrm{A} /{ }^{\circ} \mathrm{C}\right]$ & 0.002344 \\
\hline Efficiency & $\eta[\%]$ & 15,7 \\
\hline Nominal operating temperature & $\mathrm{T}_{\mathrm{N}}\left[{ }^{\circ} \mathrm{C}\right]$ & 45 \\
\hline
\end{tabular}

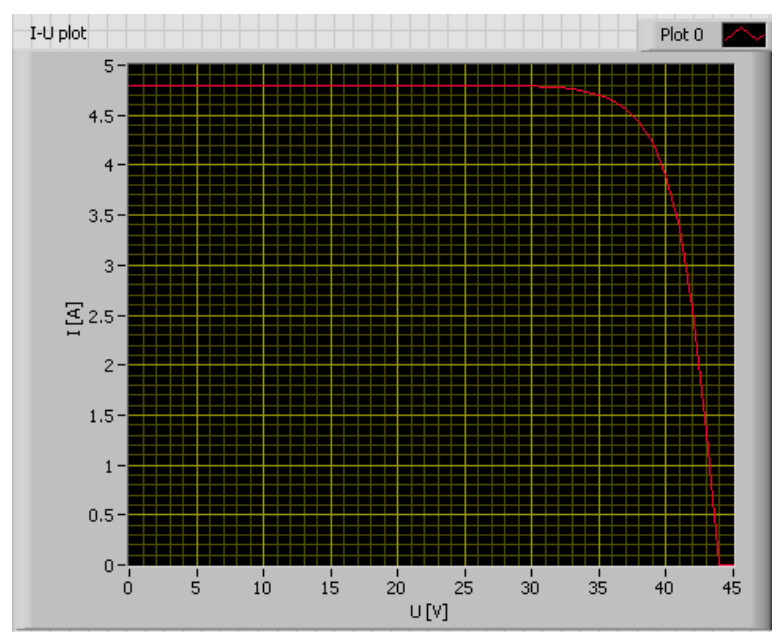

Fig. 6. Simulation results: The PV module I-U output characteristics 


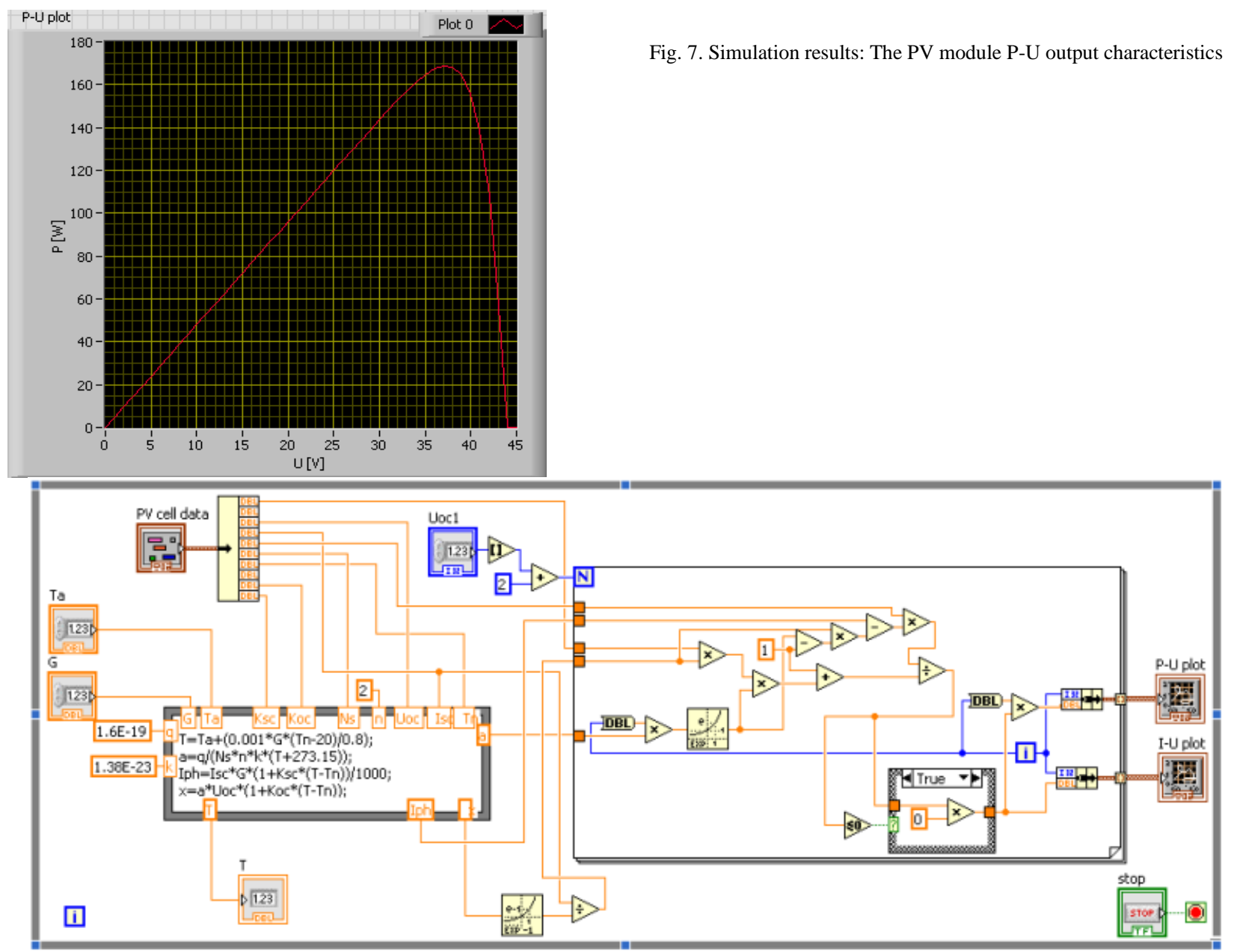

Fig. 8. A piece of the developed LabView simulation toolkit

Some LabView-based simulation results related to the PV module operation under various environmental conditions are plotted in figures 6 and 7. Of course, these plots are strongly influenced by the external conditions such as the ambient temperature $T_{A}$, or solar radiation intensity $G$. However, as it is possible to observe, the obtained results are in a good agreement with the manufacturer's provided catalogue datasheets validating the mathematical model and the LabView simulation virtual instrument. A piece of this toolkit is printed in figure 8 .

Of course there also should be mentioned that the entire simulation toolkit is still under a full development process. More accurate results set plotted under a wide range of changing environmental conditions, as well as the MPPT strategy implementation, will be provided once software implementation process turns to end.

\section{CONCLUSIONS}

The paper presents a LabView-based toolkit development processes aimed to modeling and simulate solar PV modules operation under various environmental conditions. Such a software instrument looks well suited to study and evaluate energy efficiency conversion processes in PV modules.
The first simulation results plots the I-U and P-U diagrams under different operation conditions. These curves cover the experimental ones, being in good agreement with the manufacturer's catalogue data. Future developments will be concerned to obtain more accurate results and to improve the simulation software global performances and facilities.

\section{REFERENCES}

[1] P. Hersch, "Basic photovoltaic principles and methods", United States Department for Energy, SERI/SP-290-1448, USA, Feb. 1982.

[2] R.W. Miles, K.M. Hynes, I. Forbes, "Photovoltaic solar cells: An overview of state-of-the-art cell development and environmental issues", Elsevier, $\quad$ DOI: $\quad 10.1016 /$ j.pcrysgrow.2005.10.002, www.elsevier,com/locate/pcrysgrow

[3] ST. Salmi, M. Bousgueda, A. Gastli, and A. Masmoud, "Matlab/Simulink based modelling of solar photovoltaic cell", International Journal of Renewable Energy Research, vol 2, no 2, 2012.

[4] U. Stutenbaumer, B. Mesfin, "Equivalent model of monocrystaline, polycrystaline, and amorphous silicon solar cells", Renewable Energy 18, pp. 5011-512, 1999.

[5] D. Boukoungou, Z. Koalaga, D. Njano, " Modelling and simulation of photovoltaic module considering single-diode equivalent model in Matlab", International Journal of Emerging Technology and Advanced Engineering, vol 3, issue 3, 2013, ISSN 2250-2459. 
[6] P. Srinivas, L. Kodall, V. Ramesh, "Simulation of Incremental Conductance MPPT Algorithm for PV Systems using LabView", International Journal of innovative research in Electrical, electronics, Instrumentation and Control Engineering", Vol. 4, Issue 1, January 2016, pp. 34-38.

[7] B. Velkovski, D. Pejovski, "Application of Incremental Conductance MPPT method for a photovoltaic generator in LabView", Poster $20^{\text {th }}$ International Student Conference on Electrical Engineering, $24^{\text {th }}$ May 2016, Prague, pp. 1-6.

[8] J.A. Jaleel, A.R. Omega, "Simulation on Maximum Power Point Tracking of the Photovoltaic Module using LabView", International Journal of Advanced Research in Electrical, Electronics and Instrumentation Engineering, Vol. 1, Issue 3, September 2012, ISSN 2278-8875, pp. 190-198.

[9] K.P. pradeep, C.C. Mouli, K.S. Prasad, K.Nagabhushan, "Design and Development of Optimal Controller for photovoltaic System, "International Journal of Advanced Scientific and Technical Research", Vol 1, Issue 5, February 2015, ISSN: 2249-9954, pp. 377-383.
[10] B.A. Naidu, S.A. Kumar, g.S. Reddy, "Voltage Based P\&O Algorithm for Maximum Power Point Tracking using LabView", Innovative Systems Design and Engineering, ISSN 2222-1727 (Paper), ISSN 22222871 (Online), Vol. 7, No. 5, 2016, pp. 12-16.

[11] H.S. Meyer, A.M. Soares, O.S. Neto, P.P. Prado, J.B. Goncalves, “An experimental setup to seek for maximum power point tracking of photovoltaic cells", Academic Journals, Vol. 8(47), December 2013, Doi: 10.5897/SRE2013.5738, ISSN 1992-2248. pp.2294-2297.

[12] T. Jegan, M. Kumaraguru, "LabView Based Simulator for Solar Power Plants", International Journal of Advanced Research Trends in Engineering and Technology, Vol 3, Special Issue 19, April 2016, ISSN 2394-3777 (Print), ISSN 2394-3795 (Online), pp. 129-132.

[13] H. Tian, F. Mancilla-David, K. Ellis, p. Jenkins, E. Muljadi, "Detailed Performance Model for photovoltaic Systems", Solar Energy Journal (to be published), http://www.nrel.gov/docs/fy12osti/54601.pdf. 